\title{
Modélisation de l'effet du nombre de germes sur la cavitation à bulles en paroi d'un profil et sur les pressions produites
}

\author{
L. Menoret ALSTHOM-ACB Centre d'études \& recherches de Grenoble \\ E. Blayo Ecole nationale supérieure d'informatique \& de mathématique appliquée (ENSIMAG) \\ Grenoble
}

\section{Introduction}

\subsection{Contexte}

Plusieurs études réalisées par le CERG au cours des dernières années pour les besoins de la DRET et de l'Aérospatiale, ont cherché à faire progresser la connaissance de la cavitation sur profils type aile ou ogive.

L'estimation a priori du type de cavitation produite sur un profil s'est avérée un problème souvent complexe, mettant en jeu des interactions multiples entre les différents acteurs de la cavitation :

- germes présents à l'amont du profil ;

- zone d'activation des germes située au voisinage du point de $C_{p_{\min }}$, influencée par la cavitation en aval de ce point ;

- couche limite, dont l'évolution (transition, décollement) est très liée à celle de la cavitation ;

- cavitations attachées (poches, ...), au niveau d'un décollement de couche limite ou d'une irrégularité de paroi ;

- cavités mobiles (bulles, bulles avec queue, plaques...) issues de l'activation des germes par la zone de dépression au voisinage du $C_{p_{\min }}$. La forme et la taille d'une cavité mobile sont liées à la distance du germe initial à la paroi (d'où bulle sphérique ou hémisphérique) et aux interactions avec la couche limite (d'où présence de queue), avec les autres cavités et l'anisotropie du champ de pression (d'où écart à la sphéricité) ;

- le nombre des cavités mobiles peut entraîner une suppression partielle/intermittente, ou totale/permanente d'une cavité attachée.

La progression dans l'estimation des cavitations produites et leurs effets a nécessité la réalisation d'essais sur maquette dans le tunnel hydrodynamique TH2 du CERG, ainsi que la réalisation de calculs et de modélisations. Des études sur fonds propres ont également été réalisées avec le concours de stagiaires ingénieurs.

\subsection{Cadre précis}

Il s'agit ici de présenter un enchaînement de modélisation permettant d'interpréter un enchaînement de problèmes rencontrés expérimentalement, à savoir :

(a) activation des germes par la zone de dépression et loi de similitude sur le flux de germes actifs;

(b) interaction bulle/champ de pression, et interaction bulle/bulle ;

(c) évolution moyenne d'une répartition aléatoire de bulles hémisphériques interagissantes ;

(d) pressions moyennes produites (dans le cas (c)).

\section{Activation des germes}

\subsection{Position du problème}

Parmi les germes mesurés à l'amont d'un profil, les germes actifs sont ceux qui donnent naissance à des cavités mobiles.

Modelization of the effect of the amount of germs on the cavitation with bubbles on the wall of a profile and on the pressures produced.

For a profile exhibiting a cavitation with bubbles, these bubbles will be all the more numerous, smaller and thinner when the microbubbles in the upstream liquid are also numerous. In a certain number of simplified hypotheses, an evolution model with " $N$ bubbles 》 based on the principle of apparent powers is described. This model makes it possible to assess the evolution of the bubbles' mean radius as well as the change of pressures. The results obtained are compared with tests conducted in the CERG $2 \mathrm{~m}^{3} / \mathrm{s}$ tunnel. 
L'activation d'un germe de pression critique estimée à $P_{c}=P_{\text {vap }}-\left(4 \tau / 3 R_{c}\right)$, où $\tau$ est la tension superficielle de l'eau, et $R_{c}$ le rayon critique du germe, peut se produire lorsque ce germe arrive dans une zone où la pression est inférieure à $P_{c}$.

On suppose ici que l'activation se produit à cette simple condition, c'est-à-dire qu'il n'y a pas d'effet de filtrage temporel, en lien avec le temps de réponse des germes.

L'activation du spectre de germes mesurés au granulomètre est alors due au filtrage spatial produit par la zone de dépression au voisinage du $C_{p_{\min }}$. On modélise ici la variation de pression au voisinage du $C_{p_{\min }}$ à partir de la courbure de la paroi en ce point.

La comparaison des flux de bulles obtenus par la théorie avec les flux mesurés par comptage de bulles sur photos, permet de valider une méthode pour réaliser des essais de cavitation en similitude de germes actifs.

\subsection{Champ de pression activant}

L'équation de quantité de mouvement suivant la normale à la paroi au point de $C_{p_{\min }}$ s'écrit :

$$
\frac{\partial P}{\partial y}(y)=\frac{\rho U^{2}(y)}{\mathcal{R}+y} \quad \mathcal{R}: \text { rayon de courbure } .
$$

De même, le champ de vitesse s'écrit :

$$
U(y)=V_{\infty} \sqrt{1-C_{p}(y)} .
$$

L'évolution du coefficient de pression suivant la normale au point de $C_{p_{\min }}$ est ainsi approchée, au voisinage de la paroi, par

$$
C_{p}(y)=1-\frac{1-C_{p_{\min }}}{(1+y / \mathcal{R})^{2}} .
$$

\subsection{Flux de germes pour un spectre quelconque}

Un germe de pression critique $P_{c}$ cavitera sur les lignes de courant repérées par $y$ compris entre 0 et $y_{c}$, tel que

$$
C_{p}\left(y_{c}\right)=C_{P_{c}}=\frac{P_{c}-P_{\infty}}{\frac{1}{2} \rho V_{\infty}^{2}}
$$

soit, compte tenu de l'expression $C_{p}(y)$ :

$$
y_{c}=\mathcal{R}\left(\sqrt{\frac{1-C_{P_{\min }}}{1-C_{P_{c}}}}-1\right) \text {. }
$$

Le flux de germes actifs $\phi$ (germes par seconde et par mètre transversal à l'écoulement) produit par la rencontre du spectre de germes $n_{c}$ et la zone de dépression s'obtient par l'intégrale

$$
\phi=\int_{P_{\min }}^{P_{\text {vap }}} \mathrm{d} P_{c} \int_{0}^{y_{c}} \mathrm{~d} y_{c} \cdot n_{c} \cdot u
$$

soit, après calcul :

$$
\phi=\mathcal{R} \cdot V_{\infty} \cdot \sqrt{1-C_{p_{\min }}} \int_{C_{P_{\min }}}^{-\sigma} \frac{N}{2\left(1-C_{P_{c}}\right)} \mathrm{d} C_{P_{c}} .
$$

\subsection{Cas pratique d'un spectre en puissance}

Les spectres mesurés sur le tunnel hydrodynamique TH2 du CERG se prêtent assez bien à une représentation du type

$$
N_{c}=N_{m}\left(\frac{\delta P_{c}}{\delta P_{m}}\right)^{\beta} \quad(\beta \geqslant 0)
$$

où $m$ désigne le point de pression minimale sur le profil

$$
\delta P_{m}=\left(\sigma_{m}-\sigma\right) \cdot \frac{1}{2} \rho V_{\infty}^{2}
$$

est la pression de comptage au granulomètre (sous $\left.P_{\text {vap}}\right)$.

$N_{m}$ est le résultat du comptage en $\delta P_{m}$.

L'expression du flux de germes actifs devient alors

$$
\phi_{t h}=\frac{\mathcal{R}_{n}}{2} \cdot V_{\infty} \cdot \sqrt{1+\sigma_{m}} \cdot N_{m} \cdot F_{\beta}(a)
$$

avec $a=\frac{1+\sigma}{\sigma_{m}-\sigma} \quad$ et $\quad F_{\beta}(a)=\int_{0}^{1} \frac{x^{\beta}}{a+x} \cdot \mathrm{d} x$.

Si $\beta>0$ et $a>5$ (cas pratiques), l'approximation suivante est utilisée :

$$
F_{\beta}(a) \simeq \frac{0.9\left(\sigma_{m}-\sigma\right)}{(\beta+1)(1+\sigma)} .
$$

\subsection{Comparaison avec l'expérience}

Des flux de bulles ont été mesurés d'après photos pour des essais sur quatre types d'ogives au TH2 du CERG, avec les conditions suivantes :

$$
\begin{aligned}
& \text { diamètre ogive } D=0,106 \mathrm{~m}, \\
& \text { vitesse veine } V_{\infty}=6 \text { à } 7 \mathrm{~m} / \mathrm{s}, \\
& C_{P_{\min }}=-0,4 \text { à }-0,8, \\
& \sigma=(0,5 \text { à } 1) \times\left|C_{P_{\min }}\right|,
\end{aligned}
$$

germes comptés vers $\delta P_{c}=50 \mathrm{mbar}: N_{c} \simeq 3 \mathrm{~g} / 1$ à $3 \mathrm{~g} / \mathrm{cm}^{3}$.

Il ressort que la majorité des flux mesurés sont plus forts que les flux théoriques, avec un rapport compris entre 1 et 10 . Un tel rapport est considéré comme acceptable compte tenu des incertitudes en jeu, notamment dans la détermination du spectre de germes à l'amont du profil.

\subsection{Réalisation d'essais en similitude de germes actifs}

La similitude de géométrie pour les cavités mobiles produites sur deux profils semblables observés à même $\sigma$ impose

$$
\phi \propto V_{\infty} / D^{2} .
$$

Compte tenu de la modélisation précédente de $\phi$, il apparait alors que la condition classique sur le nombre de germes actifs, comptés à 


$$
\delta P_{m}=\left(\sigma_{m}-\sigma\right) \frac{1}{2} \rho V_{\infty}^{2}: N_{m} \propto \hat{1} / D^{3}
$$

doit être assortie d'une condition de similitude sur la forme adimensionnelle des spectres actifs

$$
\frac{N_{c}}{N_{m}}=f\left(\frac{\delta P_{c}}{\delta P_{m}}\right)
$$

avec

$$
\delta P_{c}=0 \quad \text { à } \delta P_{m} \text { et } 0 \leqslant N_{c} \leqslant N_{m} .
$$

Une telle condition est sévère, et peut être assouplie, notamment lorsque les spectres $N_{c}$ sont représentables par une même forme $\left(\delta P_{c}\right)^{\beta}$ dans toute la gamme $\delta P_{c}$ utilisée.

Dans ce cas, en laissant de côté le problème de la répartition des germes actifs suivant la normale à la paroi, la similitude est obtenue plus simplement par

$$
N_{m} \propto \frac{1+\beta}{D^{3}} .
$$

La loi classique en $1 / D^{3}$ apparaît alors modulée par le facteur $(1+\beta)$ qui peut valoir de 1 à 10 environ.

\section{Interaction bulle/champ de pression et bulle/bulle}

\subsection{Position du problème}

Considérons une configuration de cavitation à bulles où un grand nombre d'entre elles peuvent, en première approximation, être considérées comme hémisphérique en paroi.

Chaque bulle $B(t)$ de rayon $R_{B}(t)$ étant alors modélisée par une source ponctuelle $q_{B}(t)=4 \pi R_{B}^{2}\left(\mathrm{~d} R_{B} / \mathrm{d} t\right)$ située en paroi, il s'agit d'obtenir l'évolution de chaque source, sous l'effet du champ de pression/vitesse $V_{0}(X)$ préexistant (sans bulles) et sous l'effet des autres bulles, notées $A(t), q_{A}(t)$.

\subsection{Interactions}

Du fait de la linéarité du problème en fluide parfait, la vitesse en un point $\vec{X}$ est la somme de la vitesse hors cavitation (avti zéro bulles), $V_{0}(\vec{X})=-\operatorname{grad} \phi_{0}(\vec{X})$, et des vitesses induites par chacune des bulles $B(t)$, $V_{B}(X, t)=-\operatorname{grad} \phi_{B}(X-B(t), t)$.

$$
\begin{gathered}
\text { Or } \quad \phi_{B}(X, t)=\frac{q_{B}(t)}{4 \pi \cdot|X-B(t)|}, \\
\text { d’où } V(X, t)=V_{0}(X)+\sum_{B} \frac{q_{B}(t)}{4 \pi} \cdot \frac{X-B(t)}{|X-B(t)|^{3}} .
\end{gathered}
$$

De plus, chaque bulle est supposée avancer avec la vitesse hors cavitation $\mathrm{d} B(t) / \mathrm{d} t=V_{0}(B(t))$.
Il reste à vérifier la conservation de la quantité de mouvement

$$
\rho \cdot \frac{\mathrm{d} V}{\mathrm{~d} t}(X, t)=-\operatorname{grad} P(X, t) .
$$

Si l'on opère comme Rayleigh dans le cas d'une bulle sphérique dans un piston (travail sur une ligne de courant allant de la paroi de bulle à l'infini) une difficulté surgit du fait de l'anisotropie du champ de pression vue par la bulle.

La méthode des puissances virtuelles permet de lisser une telle difficulté. Elle consiste à exprimer la puissance du fluide pour une variation des degrés de liberté du fluide autour des valeurs solution.

En prenant comme base de degrés de liberté les rayons des bulles, $R_{B}$, leurs variations $\delta R_{B}$ induisent une variation du champ le vitesse

$$
\delta \vec{V}=\sum_{B} \frac{\partial \vec{V}_{B}}{\partial R_{B}} \cdot \delta R_{B} .
$$

Il en résulte une puissance associée à l'équation de quantité de mouvement sur tout le volume fluide $\Omega$ :

$$
\int_{\Omega} \rho \cdot \frac{\mathrm{d} \vec{V}}{\mathrm{~d} t} \cdot \delta \vec{V} \cdot \mathrm{d} \Omega=\int_{\Omega}-\overrightarrow{\operatorname{grad}} P \cdot \delta \vec{V} \cdot \mathrm{d} \Omega .
$$

L'équation finalement obtenue, après quelques manipulations, est la suivante :

$$
\frac{3}{2}\left(\frac{\mathrm{d} R_{B}}{\mathrm{~d} t}\right)^{2}+R_{B} \cdot \frac{\mathrm{d}^{2} R_{B}}{\mathrm{~d} t^{2}}=\frac{\Delta P}{\rho}+C
$$

avec, à gauche, le terme classique d'inertie radiale, et, à droite, deux termes:

- le terme de pression, qui justifie ici la forme de Rayleigh pour un champ de pression anisotrope

$$
\frac{\Delta P}{\rho}=\frac{1}{\rho} \cdot\left(P_{\text {vap }}+\frac{K}{R_{B}^{3}}-\frac{2 \tau}{R_{B}}-P_{0}\right) .
$$

Dans cette expression ont été négligés les termes de gradient longitudinal et transversal de la pression $P_{0}$;

- le terme de couplage, $C$, qui traduit l'influence des bulles voisines sur la bulle $B$ :

$$
\begin{aligned}
C=-\sum_{A \neq B} \frac{R_{A}}{A B} \cdot\left[2 \cdot\left(\frac{\mathrm{d} R_{A}}{\mathrm{~d} t}\right)^{2}\right. & \left.+R_{A} \cdot \frac{\mathrm{d}^{2} R_{A}}{\mathrm{~d} t^{2}}\right]= \\
& =-\sum_{A \neq B} \frac{1}{4 \pi A B} \cdot \frac{\mathrm{d} q_{A}}{\mathrm{~d} t} .
\end{aligned}
$$

Dans cette expression ont été négligés les termes d'ordre supérieur en $R_{A} / R_{B}$. On suppose que l'absence de ces termes, dont la complexité est notable, ne doit pas empêcher d'obtenir une estimation du phénomène, même lorsque les bulles se touchent $\left(R_{A} \simeq R_{B} \simeq A B / 2\right)$.

Le couplage apparaît ainsi d'autant plus fort que les bulles sont proches les unes des autres, et que leur débit évolue fortement. 


\section{Evolution moyenne d'une répartition aléatoire de bulles hémisphériques interagissantes}

\subsection{Position du problème}

Les photos de cavitation sur ogive, avec injection de germes à partir d'un injecteur, révèlent une répartition aléatoire de bulles en paroi.

Il s'agit alors d'obtenir l'évolution moyenne d'une bulle en présence de ses voisines, dont la position est aléatoire, et dont la taille est la taille moyenne à la position considérée.

L'arrivée des germes dans le temps est représentée par un processus de Poisson de temps caractéristique $1 / \lambda$. La répartition transversale, qui résulte d'une dispersion par la turbulence, est supposée uniforme sur une largeur $2 a$ en paroi de profil.

Le paramètre $\lambda$ est alors déterminé par l'expression $\lambda=2 a \phi$, où $\phi$ est le flux de bulles par seconde et unité de largeur.

\subsection{Equation de l'évolution moyenne}

Pour une bulle $\mathrm{B}$, de position longitudinale $s=s_{0}$, et transversale $z=0$, seul le terme de couplage est aléatoire, et doit être moyenné sur toutes les positions possibles des bulles voisines:

$C\left(s_{0}\right)=\int_{\substack{\text { zone de } \\ \text { cavitation }}} \frac{F(s)}{B M} \cdot \mathrm{dProb}$

(Bulle en $M / 1$ Bulle en $B$ )

$\operatorname{avec} F(s)=-R\left[2 \cdot\left(\frac{\mathrm{d} R}{\mathrm{~d} t}\right)^{2}+R \cdot \frac{\mathrm{d}^{2} R}{\mathrm{~d} t^{2}}\right]$ et $R=\bar{R}(s)$.

Or le nombre moyen de bulles situées en amont de $B$ vaut :

$$
N_{1}=2 a \cdot \phi \cdot \frac{s_{0}-s(\text { début des bulles })}{V}
$$

et en aval

$$
N_{2}=2 a \cdot \phi \cdot \frac{s_{0}-s(\text { fin des bulles })}{V} .
$$

La prise en compte des lois statistiques conduit alors, après intégration de $z=-a$ à $+a$, à l'expression suivante :

$$
\begin{array}{r}
C\left(s_{0}\right)=\frac{\lambda}{a V} \cdot \sum_{i=1}^{N_{1}} \int_{s \text { debut }}^{s_{0}} F(s) \cdot \frac{\left(\frac{\lambda}{V}\right)^{i-1}}{(i-1) !} \cdot\left(s-s_{0}\right)^{i-1} \times \\
\quad \times \mathrm{e}^{-\frac{\lambda}{V} \cdot\left(s-s_{0}\right)} \times \log \frac{a+\sqrt{a^{2}+\left(s-s_{0}\right)^{2}}}{s-s_{0}} \cdot \mathrm{d} s \\
+\frac{\lambda}{a V} \sum_{i=1}^{N_{2}} \int_{s_{0}}^{s \text { fin }} F(s) \cdot \frac{\left(\frac{\lambda}{V}\right)^{i-1}}{(i-1) !} \cdot\left(s-s_{0}\right)^{i-1} \cdot \mathrm{e}^{-\frac{\lambda}{V}\left(s-s_{0}\right)} \\
\quad \times \log \frac{a+\sqrt{a^{2}+\left(s-s_{0}\right)^{2}}}{s-s_{0}} \cdot \mathrm{d} s .
\end{array}
$$

\subsection{Méthode de calcul}

Le calcul est réalisé à l'aide d'une méthode de RungeKutta appliquée aux équations

$$
\begin{aligned}
\frac{\mathrm{d} s}{\mathrm{~d} t} & =V_{0}(s) \\
\frac{\mathrm{d} R}{\mathrm{~d} t} & =U \\
\frac{\mathrm{d} U}{\mathrm{~d} t} & =\frac{1}{R}\left[-\frac{3}{2} U^{2}+\frac{\Delta P}{\rho}+C\right]
\end{aligned}
$$

dans lesquelles le terme de couplages est calculé au cycle précédent, en appelant cycle le calcul complet d'une bulle. Le cycle 0 est effectué avec un couplage nul, c'està-dire pour une bulle seule.

Le cyle commence au début du grossissement, et est arrêté peu avant le collapse, dès que la vitesse radiale atteint $\sqrt{10 . \Delta P / \rho}$ où $\Delta P$ est la pression de collapse lorsque la bulle est à son maximum.

\subsection{Comparaison avec l'expérience}

Le programme de calcul n'a, pour l'instant, été testé que sur 4 configurations de cavitation, obtenues sur ogive hémisphérique de diamètre $D=0,106 \mathrm{~m}$ à $7 \mathrm{~m} / \mathrm{s}$. Sont comparés les rayons obtenus lorsque les bulles sont à leur maximum :

\begin{tabular}{|c|c|c|c|c|c|}
\hline \multicolumn{5}{|c|}{ Expérience } & Calcul \\
\hline $\begin{array}{c}\text { N* } \\
\text { Essai }\end{array}$ & $\begin{array}{c}N b \\
\text { Photos }\end{array}$ & $\sigma$ & $\begin{array}{c}\phi \\
(b / c m / s)\end{array}$ & $R / D$ & $R / D$ \\
\hline H9 & 5 & 0,588 & 420 & 0,052 & 0,034 \\
H11 & 4 & 0,588 & 720 & 0,042 & 0,028 \\
\hline H15 & 7 & 0,466 & 530 & 0,057 & 0,051 \\
H16 & 4 & 0,466 & 920 & 0,047 & 0,051 \\
\hline
\end{tabular}

Sachant que l'injection de germes sur une ogive peut faire évoluer la taille des plus grosses bulles de $R / D=0,4$ à 0,15 dans certains cas, la présente comparaison ne semble pas infirmer le modèle. D'autres comparaisons restent cependant à réaliser pour tester le modèle.

\section{Pressions moyennes produites par une répartition aléatoire de bulles hémisphériques}

\subsection{Equation de la pression}

A partir des hypothèses et calculs précédents, l'équation de Bernouilli permet de calculer la pression en tout point, en particulier en $X$ situé en paroi :

$$
-\frac{\partial \phi}{\partial t}+\frac{V^{2}}{2}-\frac{V_{\infty}^{2}}{2}=-\frac{P-P_{\infty}}{\rho} .
$$


En négligeant les termes d'ordre supérieur en $R_{A} / A X$, il vient, lorsque $X$ est extérieur à toute bulle :

$$
\begin{aligned}
P_{\text {ext }}(X)=P_{0}(X)+\rho \sum_{A} \frac{R_{A}}{A X} \times & \\
\times & {\left[2\left(\frac{\mathrm{d} R_{A}}{\mathrm{~d} t}\right)^{2}+R_{A} \frac{\mathrm{d}^{2} R_{A}}{\mathrm{~d} t^{2}}\right] . }
\end{aligned}
$$

En moyenne le point $X$ est tantôt extérieur aux bulles, tantôt intérieur à 1 bulle, d'où la pression moyenne en $X$ :

$$
\bar{P}(x)=P_{1} \cdot P_{\text {vap }}+\left(1-P_{1}\right) \cdot \bar{P}_{\text {ext }}
$$

avec $P_{1}=$ Prob ( $M$ intérieur à une bulle).

Or, dans un processus de Poisson, la fonction de répartition des instants d'arrivée dans $(0, t)$ sachant que $N$ bulles sont arrivées dans cet intervalle, est la même que la fonction de répartition de $N$ variables aléatoires de loi uniforme sur $(0, t)$, rangées par ordre croissant.

Le calcul de $\bar{P}_{\text {ext }}$ va donc se faire en modélisant la répartition de bulles autour de $X$ comme une distribution de $N$ bulles uniforme en $S$ et $z$, d'où

$$
\bar{P}_{\text {ext }}(X)=P_{0}(X)+\frac{\phi}{V} \int \frac{F(B)}{\operatorname{Max}\left(R_{(B)},|X-B|\right)} \mathrm{d} B .
$$

Par ailleurs, $P_{1}$ est calculée par :

$$
\begin{aligned}
P_{1}= & \sum_{n=1}^{\infty} \text { Prob ( } n \text { bulles présentes) } \\
& \text { Prob }(M \text { intérieur à } 1 \text { bulle/ } n \text { bulles présentes }) \\
= & \sum_{n=1}^{\infty} \frac{(\lambda T)^{n}}{n !} \cdot \mathrm{e}^{-\lambda T} \cdot \frac{n \pi R^{2}}{\text { surface }} \\
P_{1}= & \frac{\phi}{V} \pi \cdot R^{2} .
\end{aligned}
$$

\subsection{Estimation des pressions pariétales expérimentales}

L'application du modèle en $\$ 5.1$ aux configurations de cavitation $\mathrm{H} 15$ et $\mathrm{H} 16$ du $\$ 4.4$ permet les observations suivantes :

La pression $\bar{P}(X)$ est peu affectée par la présence des bulles dans la zone où $P_{0}<P_{\text {vap. }}$. Par contre dans la zone qui suit, jusqu'au collapse des bulles, la pression avec bulles $\bar{P}(X)$ est notablement inférieure à la pression sans bulles, $P_{0}(X)$ et va jusqu'à y former un palier $\bar{P}=P_{\text {vap }}$, pour l'essai $\mathrm{H} 16$ à faible $\sigma$.

\section{Conclusion}

L'interprétation de la cavitation à bulles et de ses conséquences sur les profils passe par la mise au point de modèles théoriques.

Le premier modèle présenté ici propose une amélioration de la loi de similitude à appliquer à l'injection de germes pour obtenir une similitude géométrique de cavitation.

Le second ensemble de modélisations cherche à représenter l'évolution de nombreuses bulles et les pressions produites.

Il s'agit, dans les deux cas, de premières tentatives, qui ont le mérite d'être originales et d'exister, et qui gagneront à être testées sur d'autres configurations expérimentales.
Adresses des auteurs;

Monsieur L. Menoret Alsthom CERG Voie privée Rhône-Poulenc 38800 Le Pont de Claix Tél. : (16) 76.40.90.40

Monsieur L. Blayo 2, rue Alfred Gueymard 38400 Saint-Martin-d'Hères Tél. : (16) 76.54.56.26 
M. le Président : Je remercie MM. Menoret et BLAyo, et j'ouvre la discussion.

M. LECOFFRE: Je voudrais féliciter ces messieurs pour leur travail, et en particulier pour la qualité des résultats théoriques présentés par rapport aux résultats expérimentaux. Mais je voudrais aller un peu plus loin dans les similitudes des calculs.

Je rappellerai que la similitude en $\lambda^{3}$ est la conséquence de la similitude géométrique des écoulements sur le profil en cavitation développée. Cela s'exprime par l'égalité du $\sigma$ de cavitation d'une part et par l'égalité du nombre de bulles présentes à un instant sur le profil.

Bien entendu, la loi en $\lambda^{3}$ est une approximation de premier ordre; les spectres de germes - en modèle et en réel - ne sont jamais les mêmes dans la zone de bord d'attaque ; le comportement fin des bulles dans leur première phase de grossissement dépend de l'échelle et de la vitesse.

Egalement, on pourrait tenir compte des échelles de turbulence, des niveaux de turbulence, en amont.

La similitude complète n'est d'ailleurs pas possible du fait de ces deux derniers éléments.

Cependant, en théorie, en équilibrant les effets d'échelle sur la dynamique des bulles dans la première phase de leur grossissement, il serait nécessaire de transposer complètement les histogrammes de microbulles du modèle au prototype en créant un histogramme modèle dont les bornes des classes caractéristiques seraient en fonction de la vitesse d'écoulement, proportionnellement au carré de la vitesse, et dont le nombre de germes par classe serait proportionnel au cube de l'échelle.

Ne sachant pas réaliser pratiquement un tel histogramme, nous nous contenterons de réaliser des similitudes globalement en $\lambda^{3}$

On pourrait, de manière plus raffinée, après calcul ou mesure de la distribution des pressions sur le profil, déterminer à partir d'un histogramme réel le nombre total de bulles susceptibles d'exploser, et à partir d'une procédure analogue et d'un histogramme modèle formé de microbulles de forte pression critique, déduire les concentrations en germes qu'il est souhaitable d'avoir sur modèle pour obtenir la même géométrie sur prototype.

Tout cela est très simple...

Donc, ce travail conduit à des modélisations relativement délicates à mettre en œuvre sur une boucle d'essai en dehors des points de calcul, c'est-à-dire quand on peut avoir accès à des répartitions de pression sur la zone de bord d'attaque.

Il $\mathrm{y}$ a cependant des situations où un raffinement supplémentaire serait bienvenu, en particulier sur les modèles dont les bords d'attaque sont très petits (par exemple les modèles d'hélice)

C'était un commentaire; et je voudrais à nouveau féliciter MM. MENORET et BLAYO pour leur travail.

M. le Président : Merci M. LECOFFre pour ce long commentaire.

M. MICHEL: Je voudrais demander à M. MENOREt si le terme de couplage est important numériquement dans l'évolution d'une bulle, par rapport au terme de pression, et dans quelles conditions pratiques?

Deuxième question (en guise de commentaire): vous avez évoqué une dissymétrie de pression; cependant, d'après les films et les photos, on voit assez souvent que les bulles ont la bonne volonté de n'être pas très loin de la forme. sphérique ou hémisphérique, même en présence de gradients de pression importants. Qu'en pensez-vous?

M. MENORET: Sur le terme de couplage (M. BLAYO confirmera, je pense), surtout avant le collapse on peut dire qu'il est du même ordre que le terme de pression normal.

Sur les courbes de pression, on a pu voir que les pressions sont notablement altérées, ce qui correspond au fait que le terme de couplage est important. On voit la sensibilité finale au niveau de la pression (diapositive).
Les bulles, dans la partie de grossissement accéléré, sont encore petites, mais elles ne sont pas très couplées. Elles sont encore petites en volume. Par contre, dans la zone de grossissement inertiel et dans la région de collapse, elles occupent une grande partie du volume et elles sont très fortement couplées entre elles, d'où cette altération du champ de pression.

En ce qui concerne votre deuxième point, c'est-à-dire l'anisotropie du champ de pression, nous ne l'avons pas étudié. Notre démarche était simplement de cerner ce genre de phénomène en restant dans les hypothèses de sphéricité. Mais l'analyse théorique permet d'avoir des estimations et des ordres de grandeur. On peut saisir l'influence du gradient longitudinal de pression, en particulier dans la zone de collapse ; également l'influence des gradients transversaux, des gradients normaux à la paroi : il n'y a pas de problème, mais nous ne l'avons pas fait dans cette étude.

En ce qui concerne le commentaire de M. LECOFFRE, je voudrais aussi ajouter une remarque: $\mathrm{Si}$ l'on a réalisé des essais en similitude avec une situation réelle, on n'est peut-être pas encore totalement au point... On maîtrise les spectres de germes en ce sens qu'on sait les mesurer: dans le cadre des études qui ont été présentées on les mesure, mais on ne maitrise pas encore leur forme.

Or, on a une puissance 7 qui est obtenue sur les spectres de germes ou en tout cas des puissances assez élevées. Cela introdui des incertitudes de mesure, ce qui pour l'instant nous empêche d'aller vraiment plus loin que le premier rang du $\lambda^{3}$.

On introduit cela parce que çà vient de façon formelle, mais c'est simplement pour "faire avancer un peu le bouchon "...

M. AVELLAN: Vous avez montré que pour un prototype vitesse plus grande et dimension plus grande - on devait avoir une très faible quantité de germes. Or, malgré cette faible quantité de germes, vous avez une courbe (diapositive) qui est presque en saturation, ce qui confirme les essais que nous avons faits de notre côté.

Vous voyez que dans ce cas, si on injectait beaucoup plus de germes il ne se passerait pas grand chose; on arriverait à ce palier et on ne pourrait plus aller plus loin. Ce phénomène de saturation est vraiment important à relever dans le cadre des essais de similitude. Vous avez montré comment quantifier les niveaux de saturation.

M. HERGT: Je pense que vous avez les mêmes problèmes avec la langue allemande que moi avec la langue française... Je voudrais cependant vous demander si vous connaissez le travail de KELLER, de l'Université de Munich, concernant l'influence des bulles dans l'eau sur la cavitation.

M. MENORET: J'ai vu passer des articles signés de M. KeLLER, mais je n'ai pas eu l'occasion de les approfondir.

M. HERGT: On divise les germes en bandes de petites bulles d'air, de germes et de petits grains de sable ou de choses de ce genre.

Vous étudiez sûrement l'influence des petites bulles d'air...

\section{MENORET: Oui.}

M. HERGT: On a trouvé une différence d'influence entre les petites bulles d'air et les autres germes... Par exemple, les grains de sable portent de petites bulles d'air à la surface, mais le comportement de cet air est différent du comportement des bulles d'air libre.

M. le Président : Je crois que c'est encore un point important qui ajoute pas mal de difficultés supplémentaires aux procédés expérimentaux... On a déjả bien des difficultés avec les microbulles d'air, s'il faut encore mettre du sable !... Malheureusement c'est ce qu'on rencontre dans le cas des machines industrielles avec l'eau de nos rivières... 